Kala-azar, a term originating in Assam, India, means "black disease" and is another name for visceral leishmaniasis. It refers to the increased pigmentation on the face and trunk that accompanies the disease. The illness is characterized by intermittent fever, weight loss, splenomegaly, hepatomegaly and anemia.

\title{
Treating HIV/AIDS and leishmaniasis coinfection in Ethiopia
}

T IV/AIDS is currently recognized as the leading cause of adult illness and death in Ethiopia and has infected a cumulative total of 1500000 adults and children. Kafta Humera Woreda is an isolated area in the northwest region of the Ethiopian province of Tigray. Because of a combination of demographic and geographic factors, increased rates of both HIV transmission and visceral leishmaniasis (kala-azar) have posed serious public health challenges in the area.

Kafta Humera Woreda's position near the Eritrean and $\mathrm{Su}-$ danese borders means that it is a transit point for cross-border trade and traffic. An annual migration of male farm workers into the area causes the population to double from about 40000 during the dry season to about 80000 during the farming season. True to the model of supply and demand, the increased number of farmers is accompanied by an annual influx of commercial sex workers into the area. Therefore, both mobility and socioeconomic vulnerability are factors that contribute to increased rates of HIV transmission in the area. Moreover, the progression to AIDS is heightened by a limited access to health care.

Leishmaniasis is an opportunistic infection that is particularly troublesome for patients with HIV/AIDS in that it, too, attacks the immune system and thereby worsens a patient's already compromised ability to resist other infections. The causative organism is Leishmania, a protozoal parasite transmitted to humans through sandfly bites. Seasonal climate changes, envi$=$ ronmental degradation (through agriculture), mass movement of people (economic migration) and decreased immunity (through malnutrition) promote epidemic outbursts. Leishmaniasis infection presents in 3 forms: cutaneous, producing a large number of lesions on the face, arms and legs; mucocutaneous, causing disfiguring destruction of the mucous membranes of the nose, mouth and throat and surrounding tissues; or visceral, resulting in wasting, concurrent infections (mainly pneumonia and diarrheal disease) and bleeding secondary to thrombocytopenia and impaired liver function. In visceral leishmaniasis, the parasite infects macrophages throughout the reticuloendothelial system, compromising the immune system. Untreated, visceral leishmaniasis is uniformly fatal.

HIV/AIDS and leishmaniasis comprise a mutually reinforcing cycle of infection. Leishmaniasis accelerates the onset of AIDS by encouraging further opportunistic infections (e.g., pneumonia, tuberculosis), and thus it reduces the life expectancy of people with HIV infection. Meanwhile, HIVrelated immunosuppression increases the risk of acquiring leishmaniasis by 100-1000 times in endemic areas and encourages the development of relapsing and eventually drugresistant leishmaniasis. In a European study, $79 \%-90 \%$ of people with HIV-visceral leishmaniasis coinfection were found to have CD4 cell counts of less than $200 \times 10^{6} / \mathrm{L}$; $^{1}$ in Ethiopia, most patients die before reaching such low CD4 counts.

\section{Diagnosis and treatment}

In January 2004, Médecins Sans Frontières and the Tigray Bureau of Health initiated Ethiopia's first free HIV/AIDS treatment program. The high prevalence of visceral leishmaniasis in the area created a particularly challenging clinical reality: here, guidelines from the World Health Organization (WHO) on antiretroviral therapy in resource-limited settings need to be balanced with locally appro- priate protocols for identifying and treating HIV and visceral leishmaniasis coinfection.

In the developing world, where limited health care resources make CD4 counts difficult to obtain, low total lymphocyte counts and the presence of WHO Clinical Stage III features have been successfully used as proxies for low CD4 cell counts and viral loads in determining HIV infection and the need for antiretroviral therapy. However, in Kafta Humera Woreda, we found that clinical features of HIV/AIDS were less helpful as a guide for initiating antiretroviral therapy because they closely resemble the clinical manifestations of leishmaniasis. Moreover, visceral leishmaniasis itself reduces CD4 counts; depressed total lymphocyte and CD4 counts among coinfected patients rebound after treatment of leishmaniasis. Therefore, in coinfected patients, total lymphocyte and CD4 counts are unreliable indications for antiretroviral therapy.

Identifying the organism $L$. donovani from a tissue aspirate is the "gold standard" diagnosis for visceral leishmaniasis; however, this test is impractical to perform in a setting where the disease is highly endemic. Instead, a serologic direct agglutination test (DAT) is used as the first-line diagnostic tool.

We rely on DAT titres and clinical assessment to diagnose visceral leishmaniasis in this resource-constrained setting with a high volume of patients (between 1500 and 2000 cases per year). High DAT titres $(\geq 1: 6400)$ confirm diagnosis, and low titres $(\leq 1: 400)$ rule it out. Aspiration is performed only when DAT titres are intermediate. We have thus reduced the need for aspirate diagnosis in about $90 \%$ of cases.

The first-line drug for treating visceral leishmaniasis is sodium stibogluconate $(20 \mathrm{mg} / \mathrm{kg}$ by 
intramuscular injection for 30 days). Despite poor tolerance of this treatment among patients coinfected with HIV and visceral leishmaniasis - one-third die during treatment because of drug-induced pancreatitis ${ }^{2}$ - we use it in our program and, over the last 2 years, have seen a dramatic reduction in the rates of death caused by the disease and an increase in cure rates (Fig. 1). Other, less toxic drugs (e.g., liposomal amphotericin, miltefosine) have remained unavailable to the region.

We attribute our success in increasing cure rates and reducing death rates to the development of an efficient strategy for screening and diagnosis that allows us to identify active cases among migrant farm workers in Kafta Humera Woreda and the decentralization of diagnostic and treatment services. These factors have also led to a rise in the treatment of primary cases of visceral leishmaniasis in Kafta Humera Woreda (Fig. 2). We hope that the results from this project will result in a national policy for the diagnosis and treatment of visceral leishmaniasis and HIV coinfection in resource-limited regions that will include less toxic drug therapies.

\section{Aranka Anema}

British Columbia Centre for

Excellence in HIV/AIDS

St. Paul's Hospital

Vancouver, BC

Koert Ritmeijer

Health Adviser

Médecins Sans Frontières

Amsterdam, Netherlands

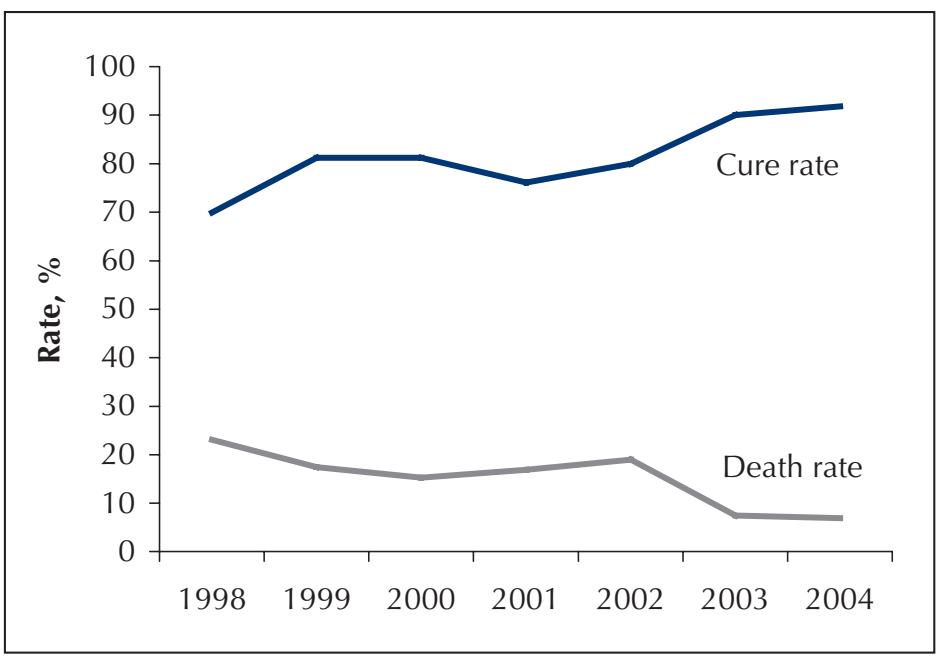

Fig. 1: Visceral leishmaniasis cure and death rates, Kafta Humera Woreda, 1998-2004.

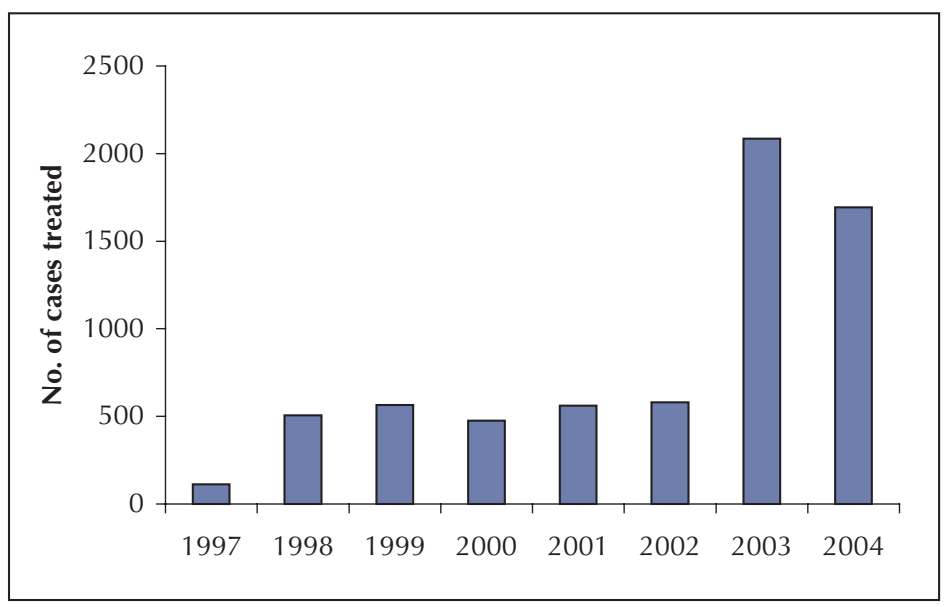

Fig. 2: Primary visceral leishmaniasis cases treated in Kafta Humera Woreda, 1997-2004.

\section{References}

1. Alvar J, Gutierrez-Solar B, Molina R, Lopez-Velez R, Garcia-Camacho A, Martinez P, et al. Prevalence of Leishmania infection among AIDS patients [letter]. Lancet 1992;339:1427.

2. Ritmeijer K, Veeken H, Melaku Y,
Leal G, Amsalu R, Seaman J, et al. Ethiopian visceral leishmaniasis: generic and proprietary sodium stibogluconate are equivalent; HIV co-infected patients have a poor outcome. Trans $\mathrm{R}$ Soc Trop Med Hyg 2001;95:668-72. 